\section{Rechnitzer S, et al.: Pretreatment Prognostic Factors for Survival in Non-Small-Cell Lung Cancer: A Multivariate Analysis of 229 Patients. Onkologie 1998;21:204-209.}

\section{Is there a Need for New Prognostic Factors in Non-Small-Cell Lung Cancer?}

In the current issue Rechnitzer et al. [1] report on prognostic factors in patients with inoperable non-small-cell lung cancer (NSCLC) undergoing palliative chemotherapy with or without radiotherapy. Twenty out of 30 clinical and laboratory parameters were found to be of prognostic value in the univariate analysis. The significant clinical factors included tumor stage, performance status, prior weight loss, and sex. Significant laboratory parameters were WBC, sodium level, alkaline phosphatase, erythrocyte sedimentation rate, LDH, GOT, GPT, albumin, gamma globulin, hemoglobin, lymphocytes, monocytes, and granulocytes. Tumor stage, sex, and serum sodium level remained significant in the multivariate analysis, indicating their independent prognostic value. The authors then developed a prognostic index based on tumor stage, sex, and serum sodium level. This index allowed to discriminate between patients with good, intermediate and poor prognosis. Similarly, a prognostic index based on 5 parameters (tumor stage, LDH, gamma-glutamyl transpeptidase, hemoglobin and lymphocyte count) was previously described by the Heidelberg group for NSCLC patients undergoing radiotherapy [2]. In general, prognostic factors are important for the patient and his/her family, for guidance of treatment, and for correct design as well as interpretation of outcome of the various treatment modalities. In NSCLC definite prognostic factors are tumor stage and performance status, and possible prognostic factors are weight loss, sex, histology, LDH, albumin, hemoglobin, platelet count, and WBC [3].

Tumor stage and performance status under consideration of pulmonary function as well as comorbidity are the major factors that currently guide treatment in patients with NSCLC. In stage I and II surgery with curative intent is the treatment of choice, and adjuvant therapies are currently evaluated within randomized clinical trials. However, despite complete tumor resection, many patients relapse. Thus, identification of the patients at high risk for relapse should be of major clinical importance because these patients might benefit from adjuvant treatment. In stage III both local and systemic therapies are required. In unresectable disease palliative chemotherapy is recommended for patients with good performance status [4]. Differences in the outcome of palliative chemotherapy might not necessarily be due to differences in the efficacy of the various chemotherapy protocols but may be caused by imbalances in known and/or still unknown prognostic factors. This possibility has particularly to be considered when comparing the outcome of the various chemotherapy protocols that include the currently available new anticancer drugs active in NSCLC. New treatment modalities probably will also require improved patient selection in the future. Thus, there is certainly a need for new factors that allow either to predict relapses in early stages after complete tumor resection or to predict survival and/or response to palliative chemotherapy in advanced NSCLC.

With regard to advanced NSCLC, the new prognostic index presented by Rechnitzer et al. [1] might be promising for NSCLC patients undergoing palliative chemotherapy, but its definite clinical usefulness will still depend on several circumstances. Firstly, the present results have to be confirmed on a large and representative patient population. Secondly, the clinical superiority in comparison to other prognostic indices remains to be demonstrated. Thirdly, biologic factors, including angiogenesis parameters, oncogenes and tumor suppressor genes, might also gain clinical importance in the future [3]. Finally, if prediction of response to chemotherapy is the major goal, drug resistance mechanisms, including alterations in oncogenes or tumor suppressor genes [5], also have to be taken into consideration.

In the meantime, it should be mandatory for all physicians involved in the treatment of NSCLC cancer patients to use the TNM system, performance status, and other factors (e.g. weight loss, comorbidity) for their treatment decisions. However, it is likely, that in the future new and more sophisticated prognostic factors will help to assign patients for the various treatment options and, thereby, improve the clinical management of patients with NSCLC.

Robert Pirker, Wien

\footnotetext{
References

1 Rechnitzer S, Bülzebruck H, Ebert W, Drings P: Pretreatment prognostic factors for survival in nonsmall-cell lung cancer: A multivariate analysis of 229 patients. Onkologie 1998;21:204-209.

2 Ohlhauser C, Bülzebruck H, Ebert W, Drings P,

Wannenmacher M: Prognostic factors for survival in inoperable non-small-cell lung cancer: A multivariate regression analysis of 456 patients with radiation therapy. Onkologie 1997;20:126-131.
}

3 Feld R, Borges M, Giner V, Ginsberg R, Harper P, Klastersky J, Lacquet L, Paesmans M, Payne D, Rosell R, Sause W, Sculier JP, Shaw E, Sorensen JB, Splinter T, Stahel R, Bunn P: Prognostic factors in non-small-cell lung cancer. Lung Cancer 1994;11 (suppl 3):S19-S123.
4 ASCO Special Article: Clinical practice guidelines for the treatment of unresectable non-small-cell lung cancer. J Clin Oncol 1997;15:2996-3018.

5 Pirker R, Krajnik G, Huber H: Cellular drug resistance in lung cancer. Onkologie 1997;20:310-314. 